\title{
Research on the Dynamic Power Control S-MAC in the Wireless Sensor Network
}

\author{
Tao Yang ${ }^{1}$, Zhenfeng $\mathrm{Qu}^{1}$ and Yong Zhang ${ }^{2, *}$ \\ ${ }^{I}$ Department of Electrical Engineering and Automation, Luoyang Institute of Science and Technology, Luoyang, Henan, \\ 471023, China \\ ${ }^{2}$ Mathematics and Computer, Chizhou University, Chizhou, Anhui, 247000, China
}

\begin{abstract}
Aiming at the Sensor-MAC (S-MAC) framework, the paper introduces the technology of controlling and adjusting the Multi-Step power. When the transmitting nodes transmit the data packets to the receiving nodes, the receiving nodes can measure the Received Signal Strength Indication, which are then transmitted back to the transmitting nodes, which is the basis of distinguishing the transmitting power. It was observed that the power of the Received Signal was larger than the threshold set at the receiving terminal. The error rates of receiving the packets can be reduced by the retransmission caused by the loss of the packets, whereas the throughput of the system can be increased. The simulated results show that the range of the throughput and the packets' successful receiving rate, which is included in the power control S-MAC as the different packet collision rate and the bounded packet re-transmission power, were increased by $3 \% \sim 10 \%$ compared with that which is not included in the power control S-MAC in Multi-hops.
\end{abstract}

Keywords: Dynamic Power Control, Wireless Sensor Network, receiving rate

\section{INTRODUCTION}

It is an important problem to determine how the devices in the Wireless Sensor Networks nodes save the energy consumption under the various environments, as the energy consumption during the communication process is the maximum $[1,2]$. Following can the mainly reasons : 1 . collision, 2. Idle Listening, 3.Overhearing, 4. Overhead. The four kinds of the reasons will be discussed in the following descriptions.

1. Collision: If there is a large amount data, it should be transmitted simultaneously within a node's transmission range, before the collision happens. The nodes in the terminal end cannot successfully receive the correct data therefore the data is lost or get erroneous. The collision makes the terminal end to re-transmit the information. The terminal end must wait for the re-transmission leading to the extra energy consumption and generation of waste [3-5].

2. Idle Listening: The nodes do not know when the data can be received so that the nodes are always in the operated state for awaiting the data transmission. If it is in the operational state without any communications, this will result in unnecessary energy consumption [6].

3. Overhearing: If the sensor nodes receive the packets of the target nodes in the non-data packet or the signal itself, or the nodes eavesdrop on the related information of the target

*Address correspondence to this author at the Mathematics and Computer, Chizhou University, Chizhou, Anhui, 247000, China;

E-mail: zhangyong@czu.edu.cn nodes in the non-data packet, the energy consumption in the sensor nodes can be caused.

4. Overhead: The energy consumption is caused by transmitting and receiving the control packets. The control packets include the RTS(Request To Send) and the CTS(Clear To Send) $[7,8]$. There is other data within the packets, but sending the control packet RTS and CTS can also cause energy consumption.

The technology of the power control is a technology of adjusting the power signal, which can measure and adjust the RSSI in the terminal end and then be transmitted back to the transmission nodes for adjusting the transmission power [9]. The RSSI of the data packet can be maintained in a certain value so that the data packet can be successfully received, and the re-transmission times and the energy consumption can be reduced $[10,11]$. The technology of the power control can measure and adjust the RSSI so that the desired effect of energy saving can be achived. The energy of the nodes in the WSN is the battery, therefore, the energy is limited and difficult to be supplied. In order to increase the life cycle of the nodes, the technology of the power control is adopted to adjust it, the energy loss of the nodes can be reduced and the throughput can be increased.

\section{THE INTRODUCTION OF THE RELATED REF- ERENCES}

S-MAC is the protocol of the active and sleeping mechanism in the fixed cycle, as shown in Fig. (1). The energy consumption in the process of listening can be reduced for the cycle of the S-MAC protocol that can make the nodes 


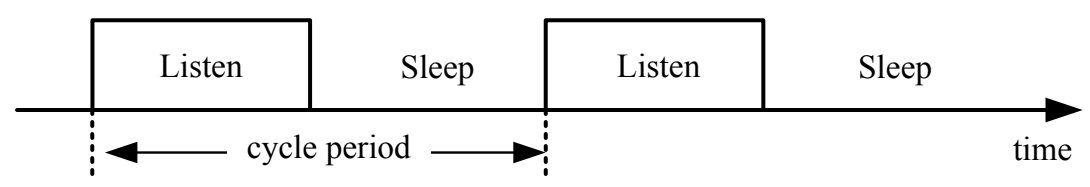

Fig. (1). The figure of the cycle mechanism in the S-MAC protocol.

enter the sleeping mechanism [12]. In addition, the nodes can have the virtual cluster which has the same active and sleeping cycle so that the synchronization among the nodes can be ensured. In this way, the energy consumption in the process of the Idle Listening can be reduced, and each node will select a schedule to exchange its neighboring node. Each node does the scheduling and establishes the scheduling table in terms of the following descriptions in the stage of the network start-up.

1. After the node monitors for a period, if other nodes' broadcast scheduling is not monitored, the monitoring node will randomly select a time to enter the sleeping state and broadcast itself scheduling. The monitoring node is called the synchronous node.

2. When the node receives the scheduling from the neighboring node, the node will follow the neighboring node's scheduling and randomly wait for a period to broadcast its scheduling. The node is called the follower.

3. When the node is the synchronous node and receives neighboring node's scheduling, the node can have two schedules.

4. S-MAC protocol adopts the physic, the virtual carrier and the exchanging mechanism with the low power RTS/CTS packets to avoid data packet collision and the overdue energy consumption during the period of the Overhearing.

Although the S-MAC is the protocol of the active and sleeping mechanism in the fixed cycle, the idle listening time is still very long and the extra energy consumption can be caused when the network flow is small. Therefore, the technology of the Multi-Step power control is adapted to adjust the RSSI in the receiving terminal and transmit it back to the transmission node, which is the basis of adjusting the transmission power. The Step Size can be adjusted according to the environment variables, to make the RSSI of the data packet maintain in the fixed value. Later, the data packet can be successfully received so that the re-transmission times and the energy consumption can be reduced and the throughput can be increased.

\section{THE INTRODUCTION OF THE POWER CON- TROL}

The paper proposes the technology of the Multi-Step power control [4], and the technology will be introduced at first. Considering the example of the RSSI, the technology of the power control can be adapted to adjust to it. $\delta$ is the Step Size with the power adjustment, $\operatorname{Pi}(\mathrm{k})$ is the value of the
RSSI in the $\mathrm{k}$ time of the $\mathrm{i}$ user, $\gamma \mathrm{i}$ is the target value demanded by the RSSI in the i user, $\Gamma \mathrm{i}(\mathrm{k})$ is the actual measured value of the RSSI in the $\mathrm{k}$ time of the $\mathrm{i}$ user and the error value erri $(\mathrm{k})=\gamma \mathrm{i}-\Gamma \mathrm{i}(\mathrm{k})$ is derived by the target value demanded by the RSSI in the $i$ user minus the actual measured value of the RSSI in the $\mathrm{k}$ time of the $\mathrm{i}$ user. The formula, showing the correlation between the Bit number of the Multi-Step and the strength multiple in the adjusted Step Size is as follows:

$2 \mathrm{~m}-1<2 \mathrm{n}+1 \leq 2 \mathrm{~m}$

in which $\mathrm{n}$ is the constant and adjusts the strength multiple of the Step Size and $m$ is the Bit number of adjusting the power control.

The Bit number of the Multi-Step and the adjusted Step Size can be computed through the formula (1), the power control $\mathrm{Pi}(\mathrm{k}+1)$ is as follows:

$\operatorname{Pi}(\mathrm{k}+1)=\Delta \mathrm{Pi}(\mathrm{k}+1)+\operatorname{Pi}(\mathrm{k}), \mathrm{Pi} \leq \mathrm{Pmax}$

in which $\operatorname{Pi}(\mathrm{k})$ is the measured power in the $\mathrm{k}$ time, $\Delta \operatorname{Pi}(\mathrm{k}+1)$ is the adjusted value in the $\mathrm{k}+1$ time, $\operatorname{Pi}(\mathrm{k}+1)$ is the actual adjusted value in the $\mathrm{k}+1$ time, and the power control $\Delta \mathrm{Pi}(\mathrm{k}+1)$ is as follows:

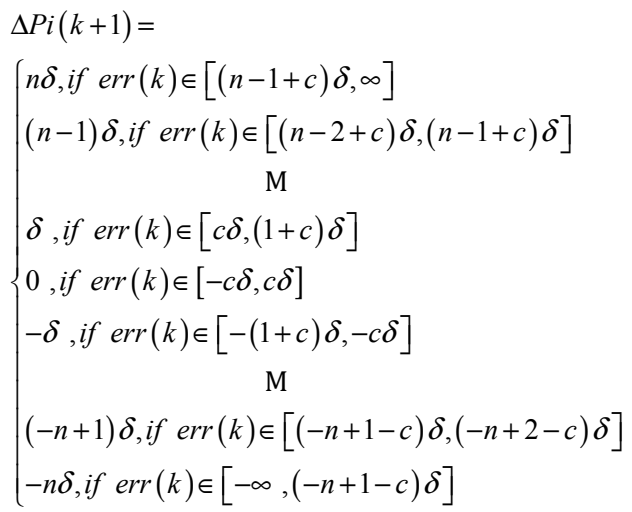

in which $\mathrm{c}$ is the fixed value which is set up as 1 .

The erri(k) value in the Multi-Step is divided into many sections, which are explained in terms of the relationship between the erri $(\mathrm{k})$ and the Step Size, as shown in the formula (3).

1. If the erri $(\mathrm{k})$ value is between the $-\delta$ and $\delta$, the measured RSSI will not do any movements.

2. If the erri(k) value is between the $\delta$ and $2 \delta$, the measured RSSI will add the $\delta$ movement.

3. If the erri $(k)$ value is between the $(n-1) \delta$ and $n \delta$, the measured RSSI will add the (n-1) $\delta$ movement. 


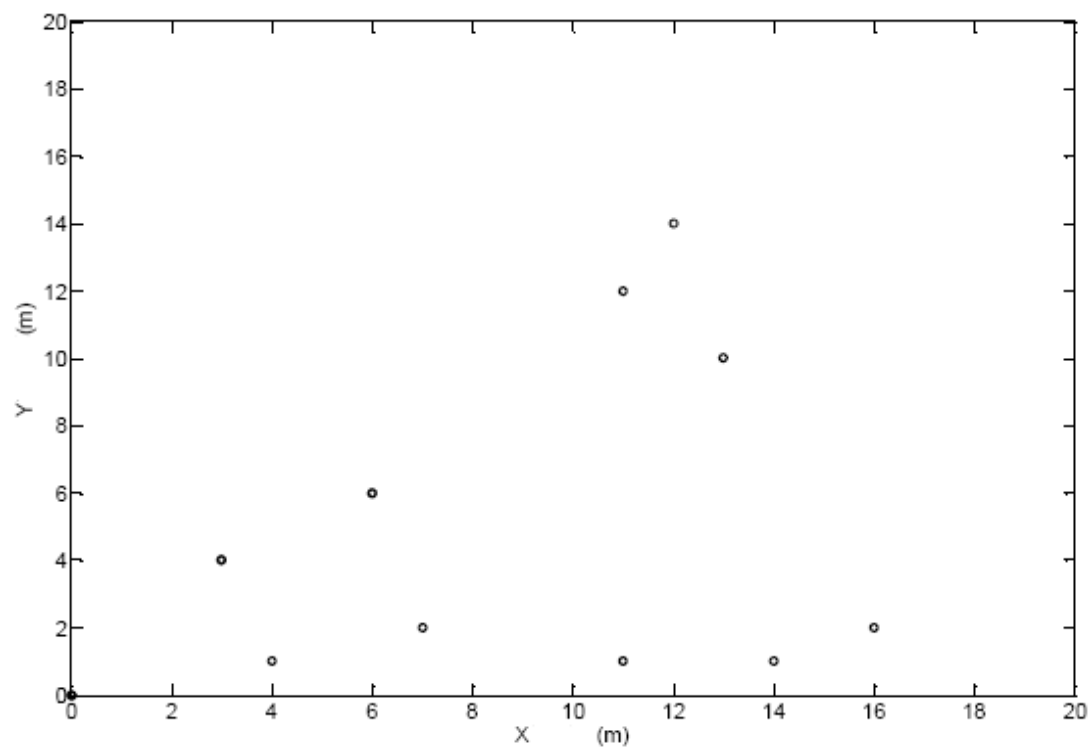

Fig. (2). Randomly deployed node.

Table 1. Cycle parameter definition table in the S-MAC activity.

\begin{tabular}{|c|c|}
\hline Parameter & Default \\
\hline \hline The node number of the sensors & 10 \\
\hline Cycle time (T) & $100 \mathrm{~ms}(0.1 \mathrm{~s})$ \\
\hline Transmission rate(R) & 20 Kbps \\
\hline Data packet length & 250 bytes \\
\hline The minimum distance among nodes & $50 \mathrm{~ms}$ \\
\hline Dormancy cycle & $0.01,0.05$ \\
\hline Collision rate & 1 \\
\hline
\end{tabular}

4. If the erri $(\mathrm{k})$ value is between the $\mathrm{n} \delta$ and $\infty$, the measured RSSI will add the $\mathrm{n} \delta$ movement.

5. If the erri(k) value is between the $-2 \delta$ and $-\delta$, the measured RSSI will minus the $\delta$ movement.

6. If the erri $(\mathrm{k})$ value is between the $-\mathrm{n} \delta$ and $(-n+1) \delta$, the measured RSSI will minus the $(-n+1) \delta$ movement.

7. If the erri(k) value is between $-\infty$ and $-n \delta$, the measured RSSI will minus the $\mathrm{n} \delta$ movement.

Although the technology of the power control can compensate the wasted energy, the power control is difficult to be compensated with a larger signal range and redundant assured energy. Therefore, the Step Size must be accorded with the following formula so that the Multi-Step can effectively adjust the value power of the RSSI to the required target region:

$\mathrm{n} \delta>|\Delta \beta|$ in which $\Delta \beta$ is a variation of the signal, and the adjusted Step Size must be larger than the variation of the signal so that the power control can reach the efficiency.

After the power control, an error range $\mid$ erri(k)| can be obtained, as shown in the following:

$|\operatorname{erri}(\mathrm{k})|<(\mathrm{n}+\mathrm{d}) \delta$ for $\mathrm{k} \geq \mathrm{K}$

\section{SYSTEM SIMULATED RESULTS}

The paper realizes the simulated results of the power control in the S-MAC protocol with the MATLAB simulation procedure. The paper simulates 10 sensor nodes which are randomly deployed in the environment whose Gaussian variable is 1.5 , and the sensor nodes are randomly deployed in the plan whose $X$ and $Y$ are $20 \mathrm{~m}$, as shown in Fig. (2). Each node can find the shortest route to transmit the data packet to the Sink, and the transmitted route nodes can divide three topology frameworks of the transmission route, the topology frameworks are as shown in Fig. (3). The simulated parameters are set up as shown in Table $\mathbf{1}$. 


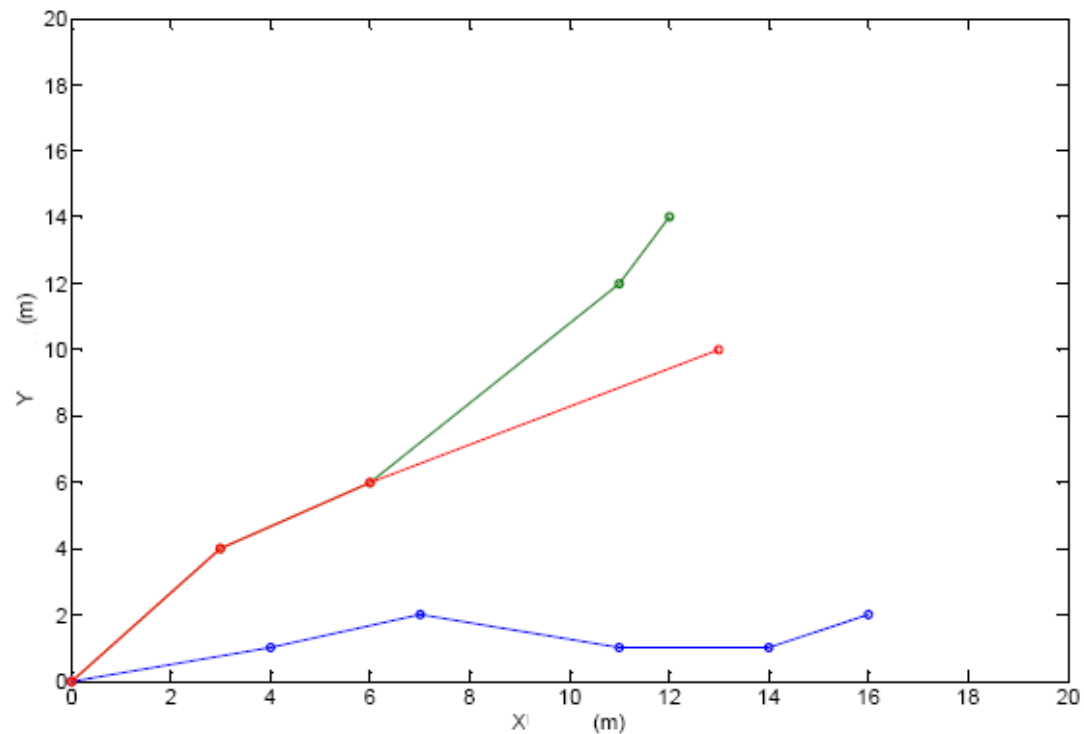

Fig. (3). The topology framework.

\subsection{The Environmental Analysis of the S-MAC which Has the Power Control}

After the RTS and the CTS packet is transmitted at the beginning of the transmission, the signal strength of the transmitted data packet during the period of transmission is set up as $-60 \mathrm{dBm}$, and the transmission terminal can measure the signal strength of the transmitted data packet during the period of transmission to the receiving terminal. The wasted energy in the transmission can be determined by the signal strength of the data packet received from the receiving terminal. The technology of the power control can be adopted to compensate the wasted energy in the next transmitted data packet. On the contrary, if there is a little difference between the signal strength of the data packet received from the receiving terminal and the signal strength of the data packet received from the transmitting terminal, the data packet in the transmission does not consume much energy so that the signal strength in the next transmission can be adjusted to reduce the energy consumption and improve energy utilization. When the power control is applied, its data packet is different from the RTS packet and CTS packet. In the case when RTS packet and CTS packet collide, the back off might be possible. If the data packet cannot finish the transmission because of a higher energy consumption, the next transmitted data packet in the transmitting terminal will increase the energy, heighten the signal strength and retransmit it immediately, while it is different from the RTS packet and CTS packet as it does not perform the back off and just needs to re-transmit it immediately after the adjustment of the power. If it does back off similar the RTS packet and CTS packet, the significance of the power control is lost. Therefore, the re-transmitted mechanism at once can avoid losing the data packet and increasing the throughput.

The formula of the Path Loss considered in the simulated environment is as follows:

$P L(d)[d B]=P L\left(d_{0}\right)[d B]+10 n \log \left[\frac{d}{d 0}\right]+X \sigma[d B]$ in which $P L\left(d_{0}\right)=20 \log \left[\frac{4 \pi d}{\lambda}\right]$ is the Path Loss of the referent distance which is assumed to be $40.0460 \mathrm{~dB}, \mathrm{n}$ is the attenuation value of the environmental parameter index which is assumed to be $2, \lambda$ is the wavelength of the carrier frequency, PL(d) is the Path Loss between the transmission and the reception, $\mathrm{X} \sigma$ is the random variation which has the Gaussian distribution whose average is 0 . The 'do' is assumed as 1.

The parameters of the power control in the S-MAC protocol in terms of formula (1) and formula (3) are as follows: Step Size $=2 、 m=3 、 n=2 、 c=1$.According to the above parameters, $\Delta \mathrm{Pi}(\mathrm{k}+1)$ in the $\mathrm{S}-\mathrm{MAC}$ protocol can be obtained, as shown in formula 7 :

$$
\Delta P_{i}(k+1)\left\{\begin{array}{l}
6 d B, \text { if } \operatorname{err}_{i}(k) \in[-76 d B m,-\infty] \\
4 d B, \text { if } \operatorname{err}_{i}(k) \in[-74 d B m,-76 d B m] \\
2 d B, \text { if } \operatorname{err}_{i}(k) \in[-72 d B m,-74 d B m] \\
0, \text { if } \operatorname{err}_{i}(k) \in[-68 d B m,-72 d B m] \\
-2 d B, \text { if } \operatorname{err}_{i}(k) \in[-66 d B m,-68 d B m] \\
-4 d B, \text { if } \operatorname{err}_{i}(k) \in[-64 d B m,-66 d B m] \\
-6 d B, \text { if } \operatorname{err}_{i}(k) \in[-64 d B m, \infty]
\end{array}\right.
$$

\subsection{The Analysis between the Throughput and the Suc- cessful Packet Received Rate:}

The chapter simulates the throughput and the successful packet received rate within the unit time under the condition of the different collided rate and the different bounded transmitted power. The simulated environment is set up as follows: The initial signal strength of the transmitting terminal is $-60 \mathrm{dBm}$, the collision rate $(\mathrm{x})$ in the S-MAC is 0.05 or 0.01 , the target value of the power strength control is $70 \mathrm{dBm}$ and the bounded transmitted power $(\mathrm{y})$ is $-90 \mathrm{dBm}$ or $-80 \mathrm{dBm}$. The average Throughput of each Hop in different 


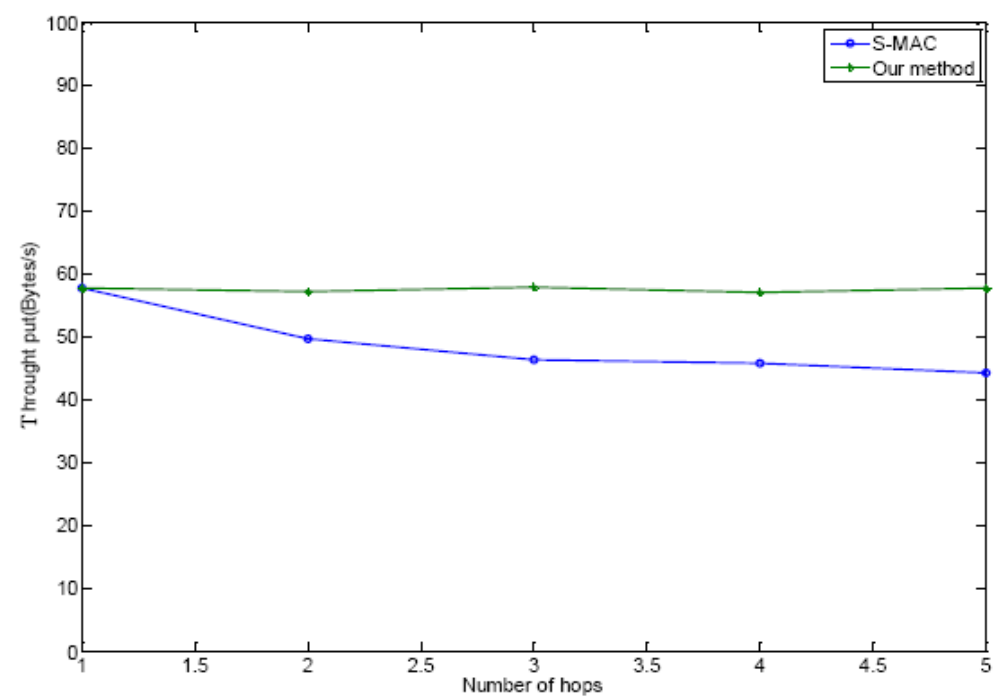

Fig. (4). The average Through-put of each Hop in the 4-Hop path.

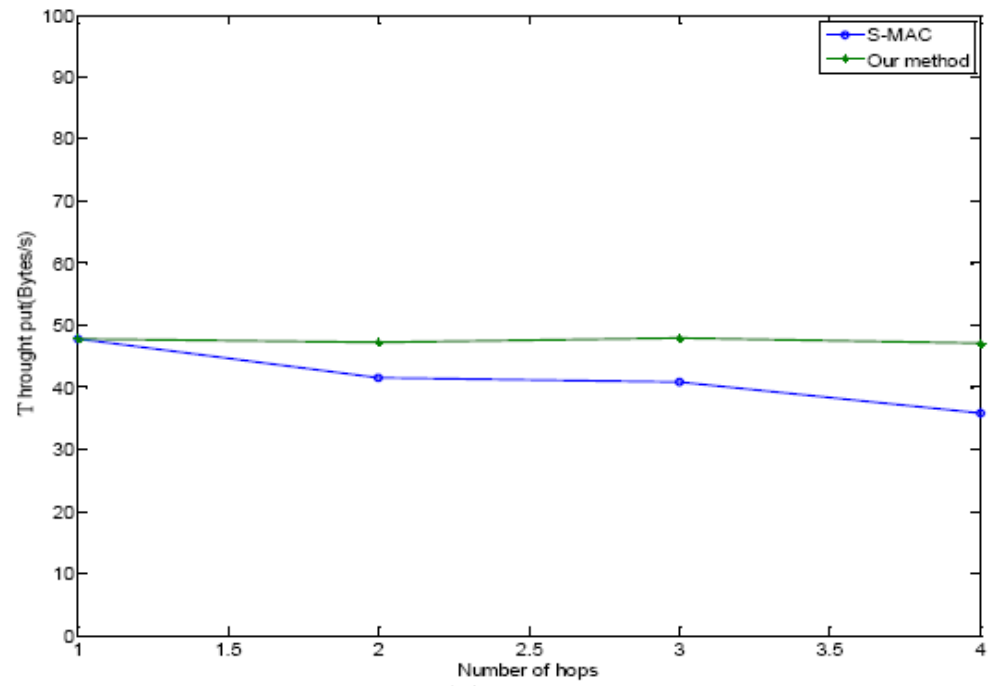

Fig. (5). The average Though-put of each Hop in the 5-Hop path.

paths obtained from transmitting 100 packets to the Sink 100 times under the condition whose collision rate in the three paths 5-Hop, 4-Hop and 3-Hop is 0.05 and the bounded transmitted power is $-90 \mathrm{dBm}$ should be simulated, and the average successful packet received rate in each path should also be simulated. Fig. (4), Fig. 5 and 6) are the simulation result of each Hop's average Throughput in the 5-Hop, 4Hop and 3-Hop, respectively.

Table $\mathbf{2}$ is the average successful packet received rate in each path. The simulated results with power control and the simulated results without power control should be compared under the condition whose collision rate is 0.01 and 0.05 , and the bounded re-transmitted power is $-80 \mathrm{dBm}$ and 90dBm.

In which $\mathrm{x}$ is the collision rate and $\mathrm{y}$ is the bounded retransmitted power.
Table 2. The average successful packet received rate.

\begin{tabular}{|c|c|c|c|c|c|c|}
\hline & $\begin{array}{c}\text { S. } \\
\text { ch } \\
\text { s. } \\
\text { hop }\end{array}$ & $\begin{array}{c}\text { s. } \\
\text { MA } \\
\text { C } \\
400\end{array}$ & $\begin{array}{c}\text { S- } \\
\text { MAa } \\
\text { C- } \\
\text { bop }\end{array}$ & $\begin{array}{l}\text { S-MLAC } \\
\text { s.hop } \\
\text { with } \\
\text { porver } \\
\text { coarrol }\end{array}$ & $\begin{array}{l}\text { S- } \\
\text { Mac } \\
\text { 4hop } \\
\text { rith } \\
\text { pormer } \\
\text { contrd }\end{array}$ & $\begin{array}{c}\text { S-MLAC } \\
\text { 3-hop } \\
\text { with } \\
\text { powex } \\
\text { eoarrol }\end{array}$ \\
\hline $\begin{array}{c}x=0.01 \\
y=- \\
s 00 \mathrm{~m} \\
x=0.0 \mathrm{~s}\end{array}$ & 0.58 & 092 & 0.98 & 096 & 0.96 & 097 \\
\hline $\begin{array}{l}y=- \\
\text { solsen }\end{array}$ & 0.57 & 09 & 0.91 & 095 & 0.94 & 094 \\
\hline $\begin{array}{c}x=001 \\
y=- \\
9005 \mathrm{~mm}\end{array}$ & 0.99 & 091 & 0.92 & 099 & 099 & 099 \\
\hline $\begin{array}{c}x=0.05 \\
y=- \\
900 \mathrm{sen}\end{array}$ & 0.58 & 09 & 0.92 & 095 & 0.95 & 095 \\
\hline
\end{tabular}




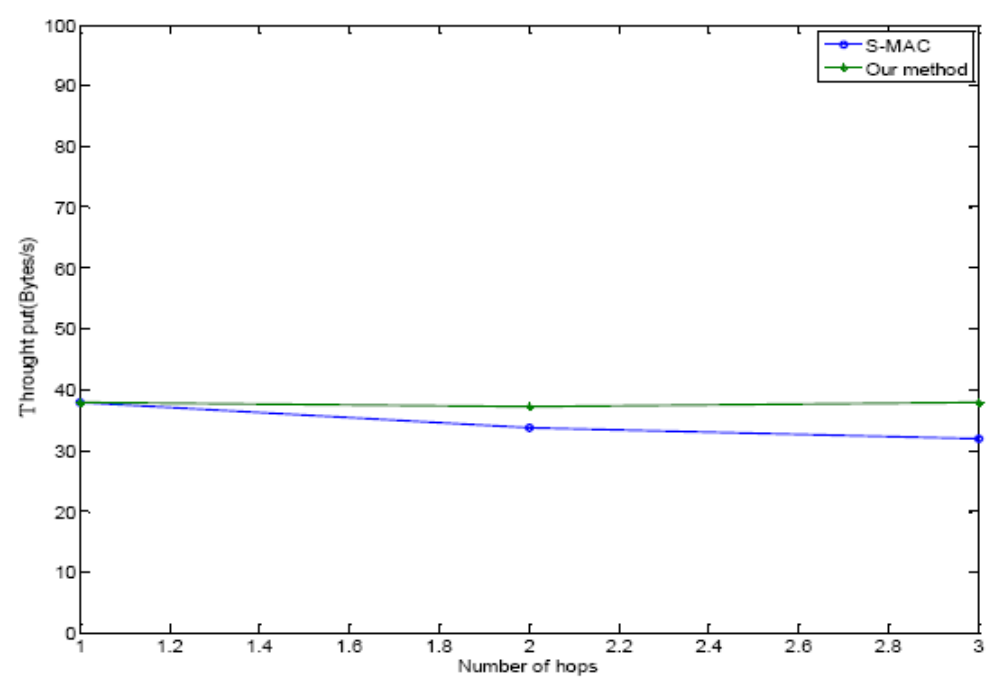

Fig. (6). The average Through-put of each Hop in the 3-Hop path.

\section{SUMMARY}

The paper does the adjustment of the power control to the received signal strength in the receiving terminal with the method of the Multi-Step power control in the transmitted terminal, and it is applied in the S-MAC. Fig. (3) shows that with the increasing number of the Hop in the transmission path, the throughput can be reduced in the S-MAC without adding the power control method, while the throughput can be maintained into a fixed value after the power control. Fig. (4) and Fig. (5) can observe the same phenomenon. Table 2 shows that the successful packet received rate cannot reach to the ideal value when the system requires that the bounded transmitted power is $-80 \mathrm{dBm}$. If the system requires that the bounded transmitted power is reduced to $-90 \mathrm{dBm}$, the successful packet received rate can reach to the ideal value. When the collision rate is 0.01 , the ideal value is 0.99 . When the collision rate is 0.05 , the ideal value is 0.95 . After the comparison, the successful packet received rate after the power control under the condition whose S-MAC is 5-hop, collision rate is 0.01 and the bounded transmitted power is $90 \mathrm{dBm}$ which can improve to maximum $10 \%$, while the successful packet received rate after the power control under the condition whose S-MAC is 53-hop, collision rate is 0.05 and the bounded transmitted power is $-90 \mathrm{dBm}$, improved to minimum 3\%. It shows that the throughput and successful data packet received rate in the S-MAC with adding the power control is larger than the throughput and the successful data packet received rate in the S-MAC without adding the power control.

The battery energy of the sensor nodes is fixed so that increasing the transmission rate and the re-transmission times can cause the whole energy consumption and the reduction of the life cycle of the sensor nodes. The above factors will be considered in the future and the method of the power control can be further researched and analyzed.

\section{CONFLICT OF INTEREST}

The authors confirm that this article content has no conflicts of interest.

\section{ACKNOWLEDGEMENTS}

Science of Technology Research of Foundation Project of Henan Province Education Department under Grant Nos.2014B520099, and Natural Science and Technology Research of Foundation Project of Henan Province Department of Science under Grant Nos. 142102210471.

\section{REFERENCES}

[1] E. Altman, K. Avrachenkov, I. Menache, G. Miller, B. J. Prabhu, and A. Shwartz, "Dynamic discrete power control in cellular networks", IEEE Transaction on Automation Control, vol. 54, no. 10, pp. 2328-2340, 2009.

[2] Q. Jian, Z. H. Gong, P. D. Zhu and C. M. Gui, "Overview of MAC protocols in wireless sensor networks," Journal of Software, vol. 19, pp. 389-403, 2008.

[3] N. Feng, S. Mau and N. B. Mandayam, "Pricing and power control for joint network-centric and user-centric radio resource management", IEEE Transactions on Communication, vol. 52, pp.1547 15572004.

[4] A. Bachir, M. Dohler, T. Watteyne, and K. K. Leung, "MAC essential for wireless sensor network," Communications Surveys \& Tutorials, IEEE, vol.12, no. 2, pp. 222-248, 2010.

[5] G. S. Ahn, S. G. Hong, E. Miluzzo, A. T. Campbell, and F. Cuomo, "Funneling-MAC: a localized, sink-oriented MAC for boosting fidelity in sensor networks," In: $4^{\text {th }} A C M$ Conference on Embedded Networked Sensor Systems (SenSys), Boulder, Colorado, USA: ACM, November 1-3, vol. 2006, pp. 293-306, 2006.

[6] H. H. Chen, Z. Fan, and J. Li, "Autonomous power control MAC protocol for mobile ad hoc networks," EURASIP Journal on Wireless Communications and Networking, vol. 6, pp. 1-10, 2006.

[7] G. S. Ahn, E. Miluzzo, and A. T. Campbell, "Demo abstract: a funneling-MAC for high performance data collection in sensor networks," In: ACM Conference on Embedded Networked Sensor Systems (SenSys). Boulder, Colorado, no. 1-3, pp. 345-346, 2006. 
[8] Y.-L. Chen, C.-P. Li, J.-W. Wang and J.-H. Wen, "Wireless systems performance analysis of multi-step power control algorithm for cellular systems," European Transaction on Telecommunications, vol. 19, pp. 193-206, 2008.

[9] J. Wen, Y. L. Chen, and J. Wang, "Convergence analysis of distributed fixed-step power control algorithm for cellular mobile systems," Wireless Communication and Mobilecomputing, vol. 8, pp. 715-731, 2008.
[10] M. I. Brownfield, “Energy-efficient Wireless Sensor Network MAC Protocol," Ph. D Dissertation, Department of Electrical Engineering, Virginia Polytechnic Institute and State University, Mar. 2006.

[11] P. Li, X. Gang and Y. Fang, "An adaptive power controlled MAC protocol for wireless Ad Hoc networks," IEEE Transactions on Wireless Communication, vol. 8, no. 1, pp. 226-233, 2009.

[12] T. V. Dam and K. Langendoen, "An adaptive energy-efficient MAC protocol for wireless sensor networks," In: ACM Sensor Systems (SenSys), Los Angeles, CA, USA, pp. 171-180, 2003.

Received: September 16, 2014

Revised: December 23, 2014

Accepted: December 31, 2014

(C) Yang et al.; Licensee Bentham Open.

This is an open access article licensed under the terms of the Creative Commons Attribution Non-Commercial License (http://creativecommons.org/licenses/bync/4.0/) which permits unrestricted, non-commercial use, distribution and reproduction in any medium, provided the work is properly cited. 